\title{
Article \\ The structural, elastic, electronic, vibrational and gravimetric hydrogen storage capacity properties of the Perovskite type Hydrides: DFT study
}

\author{
Ülkü Bayhan ${ }^{1,+} \mathbb{i} *$ and İnanç Yılmaz ${ }^{2}$ (D) \\ 1 ubayhan@mehmetakif.edu.tr \\ 2 inanc91@gmail.com \\ * Correspondence: ubayhan@mehmetakif.edu.tr \\ † Current address: Burdur Mehmet Akif Ersoy University, Department of Physics, 15030, Burdur, Turkey
}

\begin{abstract}
The structural, elastic, anisotropic elastic, electronic and vibrational properties of the Perovskite type Hydrides $\mathrm{RbXH}_{3}(\mathrm{X}=\mathrm{Be}, \mathrm{Ca}, \mathrm{Mg}$ ) were performed via Vienna $\mathrm{Ab}$ - initio Simulation Package (VASP) based on Density Functional Theory (DFT). Our results have exhibited a well-agreement with previous calculations and experiments for each compound. In order to determine physical properties of $\mathrm{RbXH}_{3}$ has been used the Generalized Gradient Approximation (GGA) with Perdew-Burke-Ernzerhof (PBE) functional at this study. Present compounds were found to be mechanically stable as well as their gravimetric hydrogen storage capacities has been investigated. The Perovskite type Hydrides $\mathrm{RbBeH}_{3}$ and $\mathrm{RbMgH}_{3}$ has an indirect bandgap of 0.274 $\mathrm{eV}$ and $2.209 \mathrm{eV}$ while $\mathrm{RbCaH}_{3}$ has a direct bandgap of $3.274 \mathrm{eV}$ respectively and therefore these compounds has shown a semiconductor behaviour at equilibrium. Besides directional dependence of anisotropic properties was visualized by representing them with maximum-minimum points.
\end{abstract}

Keywords: Hydrogen, DFT, Electronic Properties, Energy Storage, Perovskite type Hydrides

\section{Introduction}

Nowadays, the greenhouse effect and air pollution caused by the long-term high use of fossil fuels have been marked. While fossil fuels is decreased, negative impact arisen from fossil fuels has increased incrementally need for extendable and durable energy resources. For extendable and durable energy resources, there have many suggestions such as solar energy, wind energy, hydro energy etc. in literature. Among these resources Hydrogen is obviously stepped forth since Hydrogen is both clean and plentiful material in the universe and the earth. Although it has many gains, hydrogen still has not been the prime energy resource that it is very active chemically and it is too difficult to find it in pure form in the nature. Which makes possible new research areas to investigators so as to understand the nature of Hydrogen energy storage and its capabilities. That means more important than ever to look for molecules or compounds that can able to bond with hydrogen.[1-3] Furthermore the high energy ratio of hydrogen per unit mass is among the other reasons for this. In addition to, it has a wide range of usage due to next-generation solar cells and gravimetric hydrogen storage capacity. [4-6]

Firstly, Vajeeston et al. has been reported $\mathrm{MBeH}_{3}(\mathrm{M}=\mathrm{Li}, \mathrm{Na}, \mathrm{K}, \mathrm{Rb}, \mathrm{Cs})$ compounds and that study contains phase stability of present compounds. Which was represented mechanical properties such as Wyckoff position and Bulk Modulus $\left(\mathrm{B}_{0}\right)$, electronic properties such as band structure and charge density as well as estimated gravimetric hydrogen storage capacity of a monoclinic structure of $\mathrm{RbBeH}_{3}$ using VASP in this study. Also that parameter plays an important role in determining crystal structure as well[7]. In addition to the work of Vajeeston et al., the pressure dependence of elastic constants was revealed that the compound in Santosh et al. studies also shows whether $\mathrm{RbBeH}_{3}$ compound is stable in different structures[8]. 
Shinzato et al. have analyzed chemical bonding with depend on energy density analysis for $\mathrm{RbCaH}_{3}$ compound [9]. Gheboli et al., was contributed to by calculate structural, elastic, electronic and optical properties under pressure for the same compound[10]. Both works were done using CASTEP code. Another study, Vajeeston et al. have performed out mechanical properties such as Wyckoff position and Bulk Modulus $\left(\mathrm{B}_{0}\right)$, electronic properties such band structure and charge density as well as vibrational properties of cubic structure of $\mathrm{RbCaH}_{3}$ using VASP in this study [11]. As for Lamichhane et al., structural and electronic properties of $\mathrm{RbCaH}_{3}$ compound have been investigated via TB-LMTO-ASA program based on local density approximation[12]. $\mathrm{RbCaH}_{3}$ compound, one of the components of this finding, has been largely-studied compound in literature. Gingl et al., has synthesized hexagonal structure of $\mathrm{RbMgH}_{3}$ compound experimentally by sintering stoichiometric mixtures of binary hydrides at $675^{\circ} \mathrm{K}, 200$ bar $\mathrm{H}_{2}$ for 10 days [13].

In this study, structural, elastic, electronic and vibrational properties of the Perovskite type Hydrides $\mathrm{RbXH}_{3}$ (X=Be, $\mathrm{Ca}, \mathrm{Mg}$ ) compounds have been investigated. To the best of our knowledge, $\mathrm{RbBeH}_{3}$ and $\mathrm{RbMgH}_{3}$ in a cubic structure mechanical and vibrational properties are not yet represented.

\section{Calculation Methods}

The DFT calculations of $\mathrm{RbXH}_{3}(\mathrm{X}=\mathrm{Be}, \mathrm{Ca}, \mathrm{Mg}$ ) Perovskite type hydrides has been carried out Vienna Ab-initio Simulation Package (VASP) [15]. VESTA [16] software was used to visualize these structures. The electron-ion interaction with cut-off Energy of $700 \mathrm{eV}$ was taken up with the projector augmented wave (PAW) $[17,18]$ method. The Generalized Gradient Approximation (GGA) with Perdew-Burke-Ernzherhof (PBE) [19]functional was decided upon for the exchange and correlation terms in electronelectron interaction.

Kpoints sampling has been applied Gamma-centered 16x16x16 kpoints with a Methfessel-Paxton[20] smearing of $0.125 \mathrm{eV}$ for in question compounds. The Kohn-Sham equations and the Hellman-Feynmann forces have been minimized with $10^{-10} \mathrm{eV}$ and $10^{-9} \mathrm{eVA}^{-1}$, respectively. For $\mathrm{RbXH}_{3}$ compounds, cell volume and ionic positions have been relaxed by regulating ISIF $=3$ in VASP. Afterwards, in each of calculations, these achieved parameters have been used.

The stress-strain method have been used for elastic constant calculation and during that calculations have been performed by setting IBRION $=6$ and ISIF $=3$ implemented in VASP. To get an anisotropic elastic properties of compounds have been used ELATE[21] program which is an open-source software was complied from EIAM code[22].The electron configuration has been approved of $4 s^{2} 4 p^{6} 5 s^{1}$ for $R b$ atom, $2 s^{2} 2 p^{0}$ for Be atom, $3 s^{2} 3 p^{6} 4 s^{2}$ for $\mathrm{Ca}$ atom, $2 p^{6} 3 s^{2}$ for $M g$ atom and $1 s^{1}$ for $H$ atom.

The lattice dynamical properties of $\mathrm{RbXH}_{3}$ compounds were investigated linear response method through Phonopy [23] software whose method has been depended on density functional perturbation theory (DFPT)[24]. The phonon dispersion curves and density of states of $\mathrm{RbXH}_{3}$ compounds were plotted using Phonopy, in which the their unit cell of compounds has been expanded $2 \times 2 \times 2$. Thermal properties has been plotted for each compound. For calculations of lattice dynamical properties of these compounds, the ENCUT value has been chosen $500 \mathrm{eV}$ for each compound.

\section{Results and Discussion}

\subsection{Structural properties and Stability}

Generally, the Perovskite-type hydride compounds consist of a combination 1A (like $\mathrm{Li}, \mathrm{Na}, \mathrm{K}, \mathrm{Rb}$ ) or $2 \mathrm{~A}$ (like $\mathrm{Be}, \mathrm{Mg}, \mathrm{Ca}$ ) group of elements. 

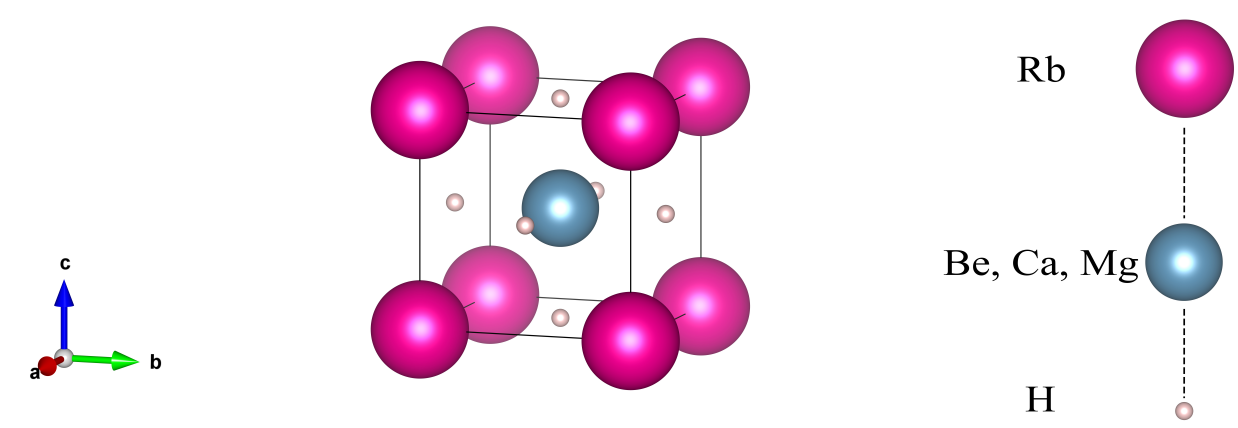

Figure 1. The crystal structure of $\mathrm{RbXH}_{3}$ compounds

$\mathrm{RbXH}_{3}$ perovskite type hydride compounds crystallize in $\mathrm{Pm} \overline{3} \mathrm{~m}$ (No:\#221) space group. Therefore, these atoms have been occupied for a $R b$ atom at $1 a(0.0,0.0,0.0)$, for an $X$ atom at $1 b(0.5,0.5,0.5)$ and for three $H$ atoms at $3 c(0.0,0.5,0.5),(0.5,0.0$, $0.5),(0.5,0.5,0.0)$ Wyckoff positions in compounds respectively. Crystal structure of the $\mathrm{RbXH}_{3}$ perovskite type compounds is represented in Figure 1. Lattice constant $(a)$, $\operatorname{volume}(V)$, density $(\rho)$, Bulk modulus $\left(B_{0}\right)$ and derivative of Bulk Modulus $\left(B_{0}{ }^{\prime}\right)$ of the calculated compounds is enumerated in Table 1., besides that results has compared with other theoretical and experimental studies. For the $\mathrm{RbXH}_{3}$ compounds, lattice constants, Bulk Modulus and derivative of Bulk Modulus were calculated using Birch-Murnaghan equation of state [25]. As is seen in Table 1., our results such as Bulk modulus, Young Modulus and Shear modulus and other properties are in harmony previous theoretical and experimental studies.

Table 1. The Lattice Parameters ( $a$ in $\AA$ ), Volumes $\left(V\right.$ in $\left.\AA^{3}\right)$, densities $\left(\rho\right.$ in $\left.g / \mathrm{cm}^{3}\right)$, Bulk Modulus $\left(B_{0}\right.$ in GPa), Derivative of Bulk Modulus $\left(B_{0}{ }^{\prime}\right.$ in GPa) and Formation Enthalpy $\left(\Delta H_{f}\right.$ in $\mathrm{eV} /$ atom $)$ of $\mathrm{RbXH}_{3}$ (X=Be, Mg and $\left.\mathrm{Ca}\right)$ compounds

\begin{tabular}{ccccccc}
\hline Compounds & $\boldsymbol{a}$ & $\boldsymbol{V}$ & $\boldsymbol{\rho}$ & $\boldsymbol{B}_{\mathbf{0}}$ & $\boldsymbol{B}_{\boldsymbol{0}}{ }^{\prime}$ & $\Delta \boldsymbol{H}_{\boldsymbol{f}}$ \\
\hline $\mathrm{RbBeH}_{3}$ & 3.760 & 53.152 & 3.046 & 48.670 & 3.729 & -0.109 \\
$\mathrm{RbMgH}_{3}$ & 4.118 & 69.858 & 2.681 & 33.365 & 3.730 & -0.618 \\
& 4.545 & & 2.275 & 24.085 & & \\
$\mathrm{RbCaH}_{3}$ & $4.547^{\mathrm{a}}$ & 93.864 & & $24.3^{\mathrm{a}}$ & 324 & -0.466 \\
& $4.5326^{\mathrm{b}}$ & & $2.29273^{\mathrm{b}}$ & $24.09^{\mathrm{b}}$ & $3.73^{\mathrm{b}}$ & \\
\hline
\end{tabular}

$[14]^{\mathrm{a}},[10]^{\mathrm{b}}$

As is seen Table 1., the raise in lattice constant makes way for diminish in the bulk modulus so that results in lessen to hardness of $\mathrm{RbXH}_{3}$ compounds. These results affirm theory for $\mathrm{RbXH}_{3}$ compounds. Because lattice constant and bulk modulus is inversely proportional on the other hand, as lattice constant increases, bulk modulus decreases. As a consequence $\mathrm{RbBeH}_{3}$ can suppose that is less compressible and harder than the other compounds. The calculated negative formation enthalpy $\left(\Delta H_{f}\right)$ with given by Eq. 1 of $\mathrm{RbXH}_{3}$ Perovskite type Hydrides are represented in Table 1 . It means that our compounds are stable as thermodynamically and synthesizable as experimantal. As is presented in Table 1., that highest stability and synthesizeability quality pertains to $\mathrm{RbMgH}_{3}$ while the lowest one $\mathrm{RbBeH}_{3}$. Since the $\mathrm{RbCaH}_{3}$ compound has been extensively studied in the literature, it is more appropriate to make comparisons about this compound than other compounds, which can be seen in Table 1. As easily understood in Table 1., it is shown that our calculations about the lattice constant were high-agreement with experimental study than the other theoretical study. Thus, it is considered that our approximation is thought to better represent the $\mathrm{RbCaH}_{3} \mathrm{compound}$. The agreement in 
bulk modulus and derivative of bulk modulus with the other theoretical work is also seen in Table 1.

The formation enthalpy has been calculated using Eq. 1.

$$
\Delta H_{f}=E_{\text {Total }}^{R b X H_{3}}-\left(E_{\text {Total }}^{R b}+E_{\text {Total }}^{X}+\left(3 \times E_{\text {Total }}^{H}\right)\right)
$$

where, $E_{\text {Total }}^{R b X H_{3}}$ indicates the total energy of $\mathrm{RbXH}_{3}$ while $E_{\text {Total }}^{R b}, E_{\text {Total }}^{X}$ and $E_{\text {Total }}^{H}$ symbolize that are lowest state energies for one atom $\mathrm{Rb}, \mathrm{X}$ and $\mathrm{H}$, respectively. To the best of our knowledge, there are no theoretical and experimental data submitted formerly for the formation enthalpy of the present compounds.
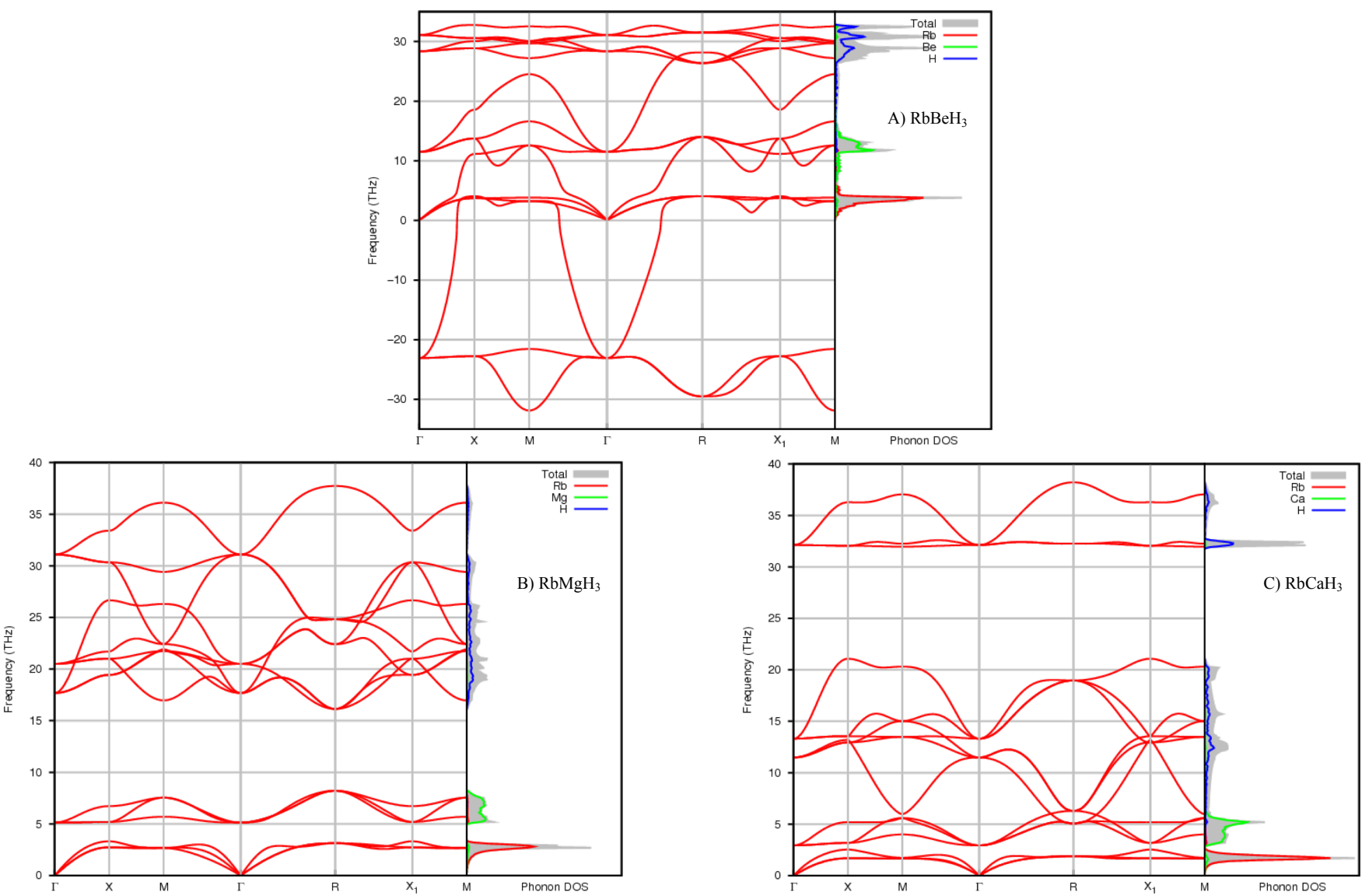

Figure 2. The Phonon Dispersion Curves and Their Corresponding Density of States for $\mathrm{RbXH}_{3}(\mathrm{X}=\mathrm{Be}, \mathrm{Mg}$, Ca) compounds A) $\mathrm{RbBeH}_{3}$, B) $\mathrm{RbMgH}_{3}$, C) $\mathrm{RbCaH}_{3}$

For $\mathrm{RbXH}_{3}$ compound, it was observed that there are including 3 acoustic and 12 optic branches totally 15 branches based on the number of atoms in the unit cell. The visualizations of these compounds about phonon plots are given by Figure 2 to be represented by Figure $2 \mathrm{~A} \mathrm{RbBeH}$, Figure $2 \mathrm{~B} \mathrm{RbMgH}$ and Figure $2 \mathrm{C} \mathrm{RbCaH}_{3}$, respectively. As is seen Figure $2 \mathrm{~A}, \mathrm{RbBeH}_{3}$ have been evaluated within this study, but it is seen that the present compound is unstable due to soft mode. That's why, the fundamental properties of $\mathrm{RbBeH}_{3}$ will be presented in order not to go out of the subject and to contribute to the literature.

Depended on Phonon dispersion curves of these atoms, it can be said that $\mathrm{RbBeH}_{3}$ is the no stable as dynamical since there was discovered soft mode, but the other two are stable dynamically since there was no observed soft mode. Phonon dispersion curves of $\mathrm{RbBeH}_{3}$ are around the $-30-30 \mathrm{THz}$ while other two are around $0-35 \mathrm{THz}$ for $\mathrm{RbMgH}_{3}$ and around $0-40 \mathrm{THz}$ for $\mathrm{RbCaH}_{3}$, respectively. As is seen these figures, it is clearly seen 
that the phonon band is split in two. This situation is a widely and expected result of ma-terials with semiconductor behaviour. In two separate regions, acoustic and optic braches have been meshed for each compound.

Because $\mathrm{RbBeH}_{3}$ has soft mode, it is unstable dynamically. For $\mathrm{RbMgH}_{3}$ at low frequencies $10 \mathrm{THz}$ below, $\mathrm{Rb}$ and $\mathrm{Mg}$ atoms are dominant due to their masses since compared $\mathrm{H}$ atom but $\mathrm{H}$ atom is dominant at high frequencies. $\mathrm{RbCaH}_{3}$ phonon dispersion curves are nested together, therefore it is seen that the existence of $\mathrm{Rb}$ and $\mathrm{Ca}$ atom around the $10 \mathrm{THz}$. Moreover, at high frequencies originate from $\mathrm{H}$ atoms as expected due to the atomic mass.

\subsection{Mechanical Properties}

The elastic constants are substantial parameters which determined to the mechanical nature of the material. For this goal, stiffness matrix of the material should be calculated. It supplies topical knowledge about rigidity, bonding nature and consistency of material which is also essential for any technological and experimental applications. The calculated elastic constants of $\mathrm{RbXH}_{3}$ compounds are enumerated in Table 2. Since the criterion of stabilise with given by Eq. 2, 3 and 4 has satisfied, our compounds are stable as mechanical[26].

Table 2. Calculated Elastic Constants $\left(C_{i j}\right.$ in GPa), Bulk Modulus, Young's Modulus, Shear Modulus ( $B, E$ and $G$ in GPa), Poisson's Ratio $(\nu)$, Universal Anisotropy Index $\left(A^{U}\right)$, Kleinman Parameter $(\xi), \mathrm{B} / \mathrm{G}, \mathrm{G} / \mathrm{B}$, Vicker's Hardness ( $H_{v}$ in GPa), longitudinal, transverse and average sound velocity $\left(V_{l}, V_{t}\right.$ and $V_{m}$ in $\left.\mathrm{m} / \mathrm{s}\right)$ and Debye Temperature $\left(\Theta_{\mathrm{D}}\right.$ in $\left.{ }^{\circ} \mathrm{K}\right)$ of $\mathrm{RbXH}_{3}$ Compounds

\begin{tabular}{|c|c|c|c|}
\hline Compounds & $\mathrm{RbBeH}_{3}$ & $\mathrm{RbMgH}_{3}$ & $\mathrm{RbCaH}_{3}$ \\
\hline$C_{11}$ & 79.252 & 62.714 & $\begin{array}{l}50.850 \\
49.51^{\mathrm{a}}\end{array}$ \\
\hline$C_{12}$ & 33.668 & 19.501 & $\begin{array}{l}10.768 \\
9.372^{\mathrm{a}}\end{array}$ \\
\hline$C_{44}$ & 67.342 & 38.893 & $\begin{array}{l}18.032 \\
18.05^{\mathrm{a}}\end{array}$ \\
\hline$B$ & 48.863 & 33.905 & $\begin{array}{l}24.129 \\
24.09^{a}\end{array}$ \\
\hline$E$ & 100.917 & 70.784 & $\begin{array}{l}44.792 \\
44.28^{\mathrm{a}}\end{array}$ \\
\hline G & 43.657 & 30.721 & $\begin{array}{c}18.810 \\
18.8^{\mathrm{a}}\end{array}$ \\
\hline$\nu$ & 0.156 & 0.152 & $\begin{array}{l}0.191 \\
0.17^{\mathrm{a}}\end{array}$ \\
\hline$A^{U}$ & 1.552 & 0.427 & 0.013 \\
\hline$\xi$ & 0.560 & 0.458 & $\begin{array}{l}0.363 \\
0.34^{\mathrm{a}}\end{array}$ \\
\hline$B / G$ & 1.119 & 1.104 & 1.283 \\
\hline$G / B$ & 0.894 & 0.906 & 0.779 \\
\hline$H_{\nu}$ & 12.854 & 10.130 & 5.278 \\
\hline$V_{l}$ & 5958.758 & 5284.217 & $\begin{array}{l}4651.374 \\
4569.07^{\mathrm{a}}\end{array}$ \\
\hline$V_{t}$ & 3785.763 & 3384.963 & $\begin{array}{l}2875.793 \\
2866.15^{a}\end{array}$ \\
\hline$V_{m}$ & 4160.367 & 3718.564 & $\begin{array}{l}3171.653 \\
3156.09^{\mathrm{a}}\end{array}$ \\
\hline$\Theta_{D}$ & 563.328 & 459.659 & $\begin{array}{l}355.291 \\
281.04^{\mathrm{a}}\end{array}$ \\
\hline
\end{tabular}




$$
\begin{array}{rlrl}
\left(\frac{C_{11}-2 C_{12}}{3}\right) & >0 ; & \left(\frac{C_{11}-C_{12}}{2}\right) & >0 \\
C_{44} & >0 ; & B & >0 \\
C_{12}<B & <C_{11} &
\end{array}
$$

As is seen Table 2., since $C_{11}$ value is higher than $C_{44}$ value for each compound, our compounds are probable to adventure pure shear distortion when considering resistance to uniaxial compression. The pure shear distortion is defined by $\mathrm{C}_{44}$ on (100) crystal plane and so based on $\mathrm{C}_{44}$ data, $\mathrm{RbBeH}_{3}$ is harder than $\mathrm{RbMgH}_{3}$ and $\mathrm{RbCaH}_{3}$ but lowest one is $\mathrm{RbCaH}_{3}$. This result confirms the predictions with regard to hardness made in the previous section.

The directionality of any compound, i.e., the degree of anisotropy, is defined by the elastic anisotropy factor given by the formula for cubic crystals in Eq. 5, which is also known as the Universal Elastic Anisotropy Index $\left(A^{U}\right)$ [27]. This value is 0 for isotropic crystals. A greater $A^{U}$ value shows directionality of the compound.

$$
A^{U}=\frac{6}{5}\left(\sqrt{A}+\frac{1}{\sqrt{2}}\right)^{2} \quad A=\frac{G_{V}}{G_{R}}
$$

In this study, bulk, shear, Young's modulus and Poissons's Ratio have been calculated using Voigh-Reuss-Hill (VRH) approximation and and it is given by Eq. 6 and 7, respectively as well as calculated results is listed in Table 2.

$$
\begin{aligned}
B & =\frac{C_{11}+2 C_{12}}{3} ; & G & =\frac{G_{V}}{G_{R}} \\
E & =\frac{9 B G}{3 B+G} ; & \nu & =\frac{3 B-2 G}{2(3 B+G)}
\end{aligned}
$$

In addition to that parameters the other parameters such as Kleinman parameter, B/G ratio, G/B ratio called Pugh's modulus and Vicker's hardness parameters presented by Table 2 as well as their equations are also given by Eq. 8 and Eq. 9 , respectively. When $\mathrm{B}_{0}$ values in Table 1 and $\mathrm{B}$ values in Table 2 are compared, it is seen that study is executed with good-precision. Because there is a little deviation between compared values. As is seen Table 2 the bulk modulus is a measure of the resistance of the compound to volume change under hydrostatic pressure. Among these compounds $\mathrm{RbBeH}_{3}$ is less compressible compared with $\mathrm{RbMgH}_{3}$ and $\mathrm{RbCaH}_{3}$. E is accepted that the compound is an indicator of degree of hardness and $\mathrm{G}$ is accepted that it is a measure of the impedance to plastic distortion of the compound. Using their calculated data, it can be said that among these structures $\mathrm{RbBeH}_{3}$ is harder compared to $\mathrm{RbMgH}_{3}$ and $\mathrm{RbCaH}_{3}$.

$$
\begin{aligned}
\xi & =\frac{C_{11}+8 C_{12}}{7 C_{11}+2 C_{12}} \\
H_{v} & =2\left(k^{2} G\right)^{0.585} \quad ; k=G / B
\end{aligned}
$$

There are many suggestions in the literature to predict whether a material ductile or brittle. The most common of these is look at the $B / G$ ratio. The $B / G$ ratio of material less than 1.75 , it is considered brittle and if it is large, it is considered ductile. In addition to, according to the widely known Frantsevich rule[28], Poisson's ratio $(\nu)$ has a vital value of 0.26 . If that value of the material greater than 0.26 material is ductile, if it is smaller than 0.26 material is brittle. According to these two rules our compounds are brittle. The Pugh's modulus and $\nu$ are used to define the bonding nature of the material. The point is here that if Pugh's modulus is around 1.1, covalent bonding character is do-minant 
in material, if Pugh's modulus is around 0.6, ionic bonding character is dominant in the material. Similarly, it is possible to explain that situation with $\nu$. If $\nu$ is around 0.25 , the ionic bonding is dominant in the material, if $\nu$ is around 0.1 , the covalent bonding is dominant in the material. The outcome of Pugh's modulus ratio has been found for $\mathrm{RbBeH}_{3}, \mathrm{RbMgH}_{3}$ and $\mathrm{RbCaH}_{3} 0.894,0.906$ and 0.779 , respectively, besides that the $\checkmark$ has been found for same materials $0.156,0.152$ and 0.191 , respectively. Concerning about the present case, it can be said that there are both covalent and ionic contribution in our materials. It is seen that this result is to be coherent previously theoretical and experimental studies.

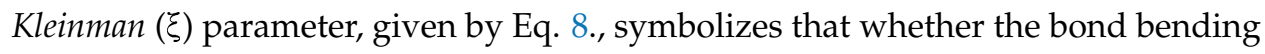
or the bond stretching in the compound. When there was a bond bending in the compound, Kleinman parameter is minimised or null otherwise while there was a bond stretching in the compound, Kleinman parameter is close to 1. As is seen Table 2., The calculated $\xi$ value is $0.560,0.458$ and 0.363 for $\mathrm{RbBeH}_{3}, \mathrm{RbMgH}_{3}$ and $\mathrm{RbCaH}_{3}$, respectively. It is seen that the compounds are impedance to bond bending for each but among them $\mathrm{RbBeH}_{3}$ also appears to have the highest impedance.

Vicker's $\left(H_{v}\right)$ hardness is calculated in question compounds. As is known Vicker's hardness give information to us related to the hardness of the material. As is seen in Table 2, Vicker's hardness was calculated as Chen et al.[29]. in this work using Eq. 9. So, these materials are comparatively hard materials as both calculated Vicker's hardness values are below the super hardness limit as $40 \mathrm{GPa}$.

$$
\begin{aligned}
v_{t} & =\sqrt{\frac{G}{\rho}} \\
v_{l} & =\sqrt{\frac{3 B+4 G}{3 \rho}} \\
v_{m} & =\left[\frac{1}{3}\left(\frac{2}{v_{t}^{3}}+\frac{1}{v_{l}^{3}}\right)\right]^{-\frac{1}{3}} \\
\Theta_{D} & =\frac{h}{k_{b}}\left[\frac{3 n}{4 \pi}\left(\frac{N_{A \rho}}{M}\right)\right]^{\frac{1}{3}} \times v_{m}
\end{aligned}
$$

In Eq. $13, h$ is Planck constant, $k_{b}$ is Boltzmann constant, $\rho$ is a density, $n$ is the number of atoms in the unit cell, $N_{A}$ is Avogadro's number, $M$ is a molecular weight and $v_{m}$ is an averaged of the sound velocities calculated using $v_{l}$ and $v_{t}$. The Debye temperature represents the temperature obtained when the unit of wavelength of the phonon frequency is equal to the length of the cell, which gives information about some important and distinguish physical properties of solids such as specific heat and melting temperature. For understood about relationship solids and temperature, Debye temperature should be calculated by using the elastic constants called Navier equations. That equation is given by Eq. 10, 11, 12 and 13 respectively. Debye temperature and longitudinal, transverse and average sound velocity of these compounds are viewed in Table 2. According to the presented data in Table 2, sound velocities and Debye temperatures decrease with a unit cell lenght of theses compounds increases. From that results there was a powerful dependence of the phonon frequencies on the unit cell volume. From that findings, it can be concluded that there was a powerful dependence between the their unit cell volumes and phonon frequencies.

\subsection{Anisotropic Properties}

Unaccustomed phonon modes, anisotropic plastic distortion and similar some deformations can be caused by anisotropy of elasticity of the material. That's why, the 
anisotropy of elasticity has quite important playing role of technological and experimental applications obviously. Furthermore to increase the mechanical resistance of a material, anisotropic properties of material must be understood and as well it is also intended to bring up of direction dependence of a material. In this study, anisotropic properties of $\mathrm{RbXH}_{3}$ compounds represent by $2 \mathrm{D}$ and $3 \mathrm{D}$ visualization and their maximum and minimum thanks to ELATE. The maximum and minimum values of the $\mathrm{RbXH}_{3}$ compounds are enumerated in Table 3 as well as their visualizations are viewed in Figure 3 to be represented by Figure 3A linear compressibility, Figure 3B Poisson's Ratio, Figure 3C Shear Modulus and Figure 3D Young's Modulus of $\mathrm{RbCaH}_{3}$, respectively. Following visualizations presented belong to $\mathrm{RbCaH}_{3}$. Others are similar and it is also shown in Appendix A, Figure 1 and 2.

Table 3. Minimum and Maximum points of Young's Modulus ( $E_{\min }$ and $E_{\max }$ in GPa), Linear Compressibility $\left(\beta_{\min }\right.$ and $\beta_{\max }$ in TPa $\left.{ }^{-1}\right)$, Shear Modulus $\left(G_{\min }\right.$ and $G_{\max }$ in GPa) and Poisson's Ratio $\left(\nu_{\min }\right.$ and $\left.\nu_{\max }\right)$ for $\mathrm{RbXH}_{3}$ Compounds

\begin{tabular}{ccccccccc}
\hline \multirow{2}{*}{ Compounds } & \multicolumn{2}{c}{ Young's Modulus } & \multicolumn{2}{c}{ Linear Compressibility } & \multicolumn{2}{c}{ Shear Modulus } & \multicolumn{2}{c}{ Poisson's Ratio } \\
& $\boldsymbol{E}_{\text {min }}$ & $\boldsymbol{E}_{\text {max }}$ & $\beta_{\text {min }}$ & $\beta_{\text {min }}$ & $\boldsymbol{G}_{\text {min }}$ & $\boldsymbol{G}_{\text {max }}$ & $\boldsymbol{P}_{\text {min }}$ & $\boldsymbol{P}_{\text {min }}$ \\
\hline $\mathrm{RbBeH}_{3}$ & 59.175 & 138.43 & 6.822 & 6.822 & 22.792 & 67.342 & $\mathbf{- 0 . 2 3}$ & 0.523 \\
$\mathrm{RbMgH}_{3}$ & 53.463 & 84.405 & 9.831 & 9.831 & 21.607 & 38.893 & -0.052 & 0.327 \\
$\mathrm{RbCaH}_{3}$ & 43.308 & 47.086 & 13.815 & 13.815 & 18.032 & 20.041 & 0.164 & 0.225 \\
\hline
\end{tabular}

As is known, the Poisson's Ratio is a measure of the distortion of a material. The maximum value is 0.5 while the minimum value of the Poisson's Ratio is -1 . Since the maximum Poisson's Ratio value ( $\nu_{\max }$ ) of $\mathrm{RbBeH}_{3}$ falls outside that limit, it is thought that the deformation will be permanent for this material. Also, these results shown that high pressure, investigate is required for this material.
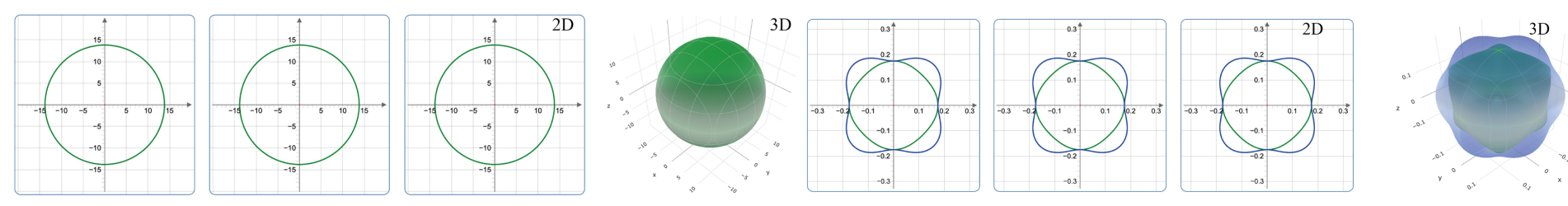

A) Linear Compressibility of $\mathrm{RbCaH}_{3}$
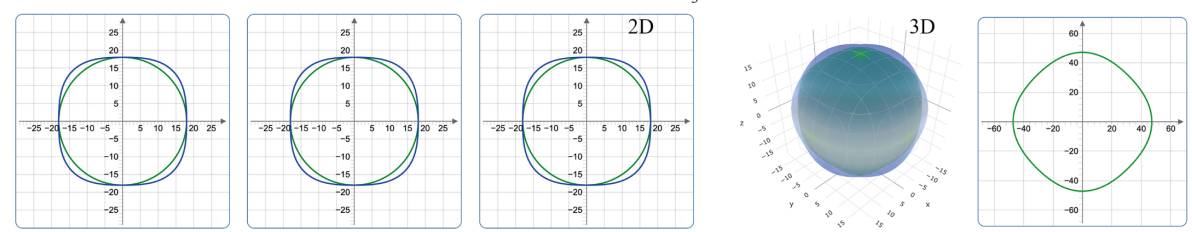

B) Poisson's Ratio of $\mathrm{RbCaH}_{3}$

C) Shear Modulus of $\mathrm{RbCaH}_{3}$
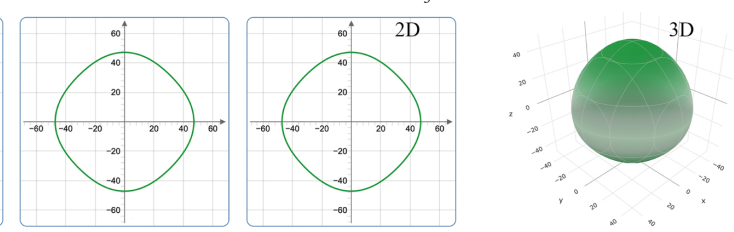

D) Young Modulus of $\mathrm{RbCaH}_{3}$

Figure 3. A) Linear Compressibility, B) Poisson's Ratio, C) Shear Modulus and D) Young's Modulus of $\mathrm{RbCaH}_{3}$

\subsection{Electronic Properties}

Electronic band structures and their corresponding density of states(DOS) have been used to explain electronic behaviours of any compounds. In this study, electronic calculations of $\mathrm{RbXH}_{3}$ compounds were accomplished using VASP along with first Brillouin zone with referencing high symmetry points of these compounds and their outcomes are given by Figure 4, to be represented by Figure $4 \mathrm{~A} \mathrm{RbBeH}_{3}$, Figure $4 \mathrm{~B}$ $\mathrm{RbMgH}_{3}$ and Figure $4 \mathrm{C} \mathrm{RbCaH}_{3}$, respectively. 

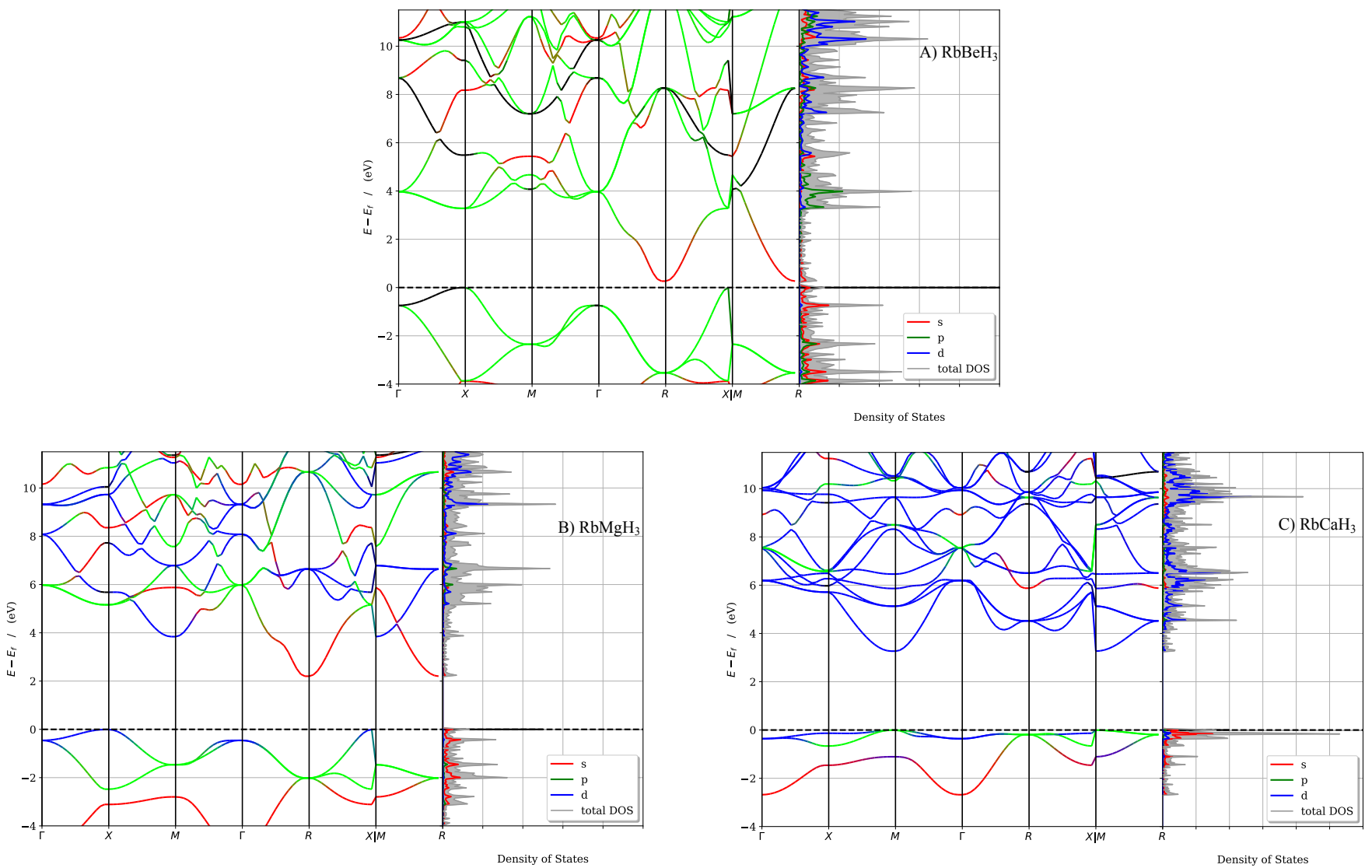

Figure 4. A) $\mathrm{RbBeH}_{3}$ Band Structure and corresponding Density of States(DOS), B) $\mathrm{RbMgH}_{3}$ Band Structure and corresponding Density of States(DOS), C) $\mathrm{RbCaH}_{3}$ Band Structure and corresponding Density of States(DOS)

The visualizations following are examined, it can be seen from Figure 4 that the investigated compounds show semiconductor behavior. $\mathrm{RbBeH}_{3}$ and $\mathrm{RbMgH}_{3}$ have an indirect bandgap as 0.274 and $2.209 \mathrm{eV}$, respectively, while $\mathrm{RbCaH}_{3}$ has a direct bandgap as $3.274 \mathrm{eV}$. For $\mathrm{RbCaH}_{3}$, Ghebouli et al. has reported the same value as $3.27 \mathrm{eV}$ for the same compound[10]. That result exhibits how compatible our research is with other theoretical and experimental studies. As is seen in Figure 5 for compounds with indirect bandgap, $\mathrm{H}$ atoms dominate near the Fermi level.

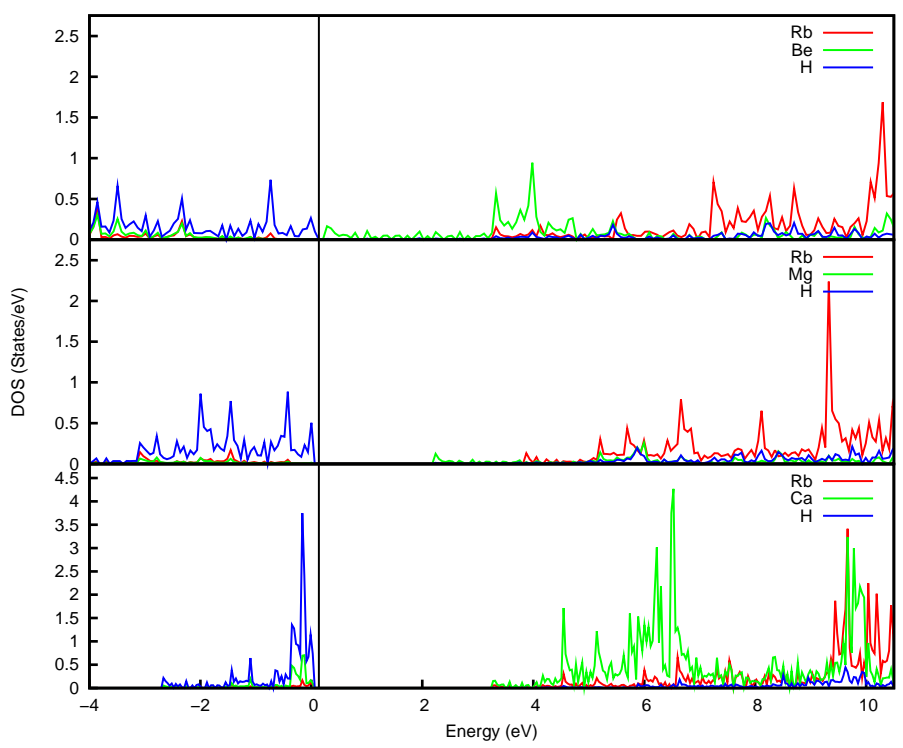

Figure 5. Partial DOS for $\mathrm{RbXH}_{3}$ Compounds 


\subsection{Thermal Properties}

For the thermal properties of these compounds, it was used in quasi-harmonic approximation to determined of their thermodynamic properties such as Helmholtz free energy, enthalpy, entropy, and constant volume heat capacity in 0 to $2000{ }^{\circ} \mathrm{K}$ temperature range for $\mathrm{RbMgH}_{3}$ and $\mathrm{RbCaH}_{3}$. Figure $6 \mathrm{~A}$ and Figure $6 \mathrm{~B}$ indicate free energy, entropy, heat capacity and energy as a function of temperature, respectively. Throughout, the temperature 0 to $2000^{\circ} \mathrm{K}$, it can be obviously seen that free energy was decreased while entropy and energy was increased besides that the heat capacity reaches a constant called as Dulong-Petit limit around the $700{ }^{\circ} \mathrm{K}$.

A) $\mathrm{RbMgH}_{3}$

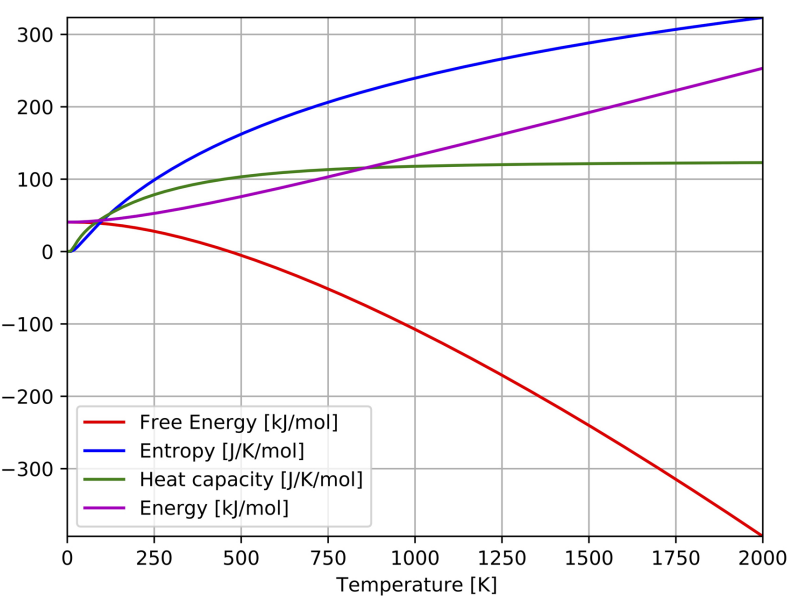

B) $\mathrm{RbCaH}_{3}$

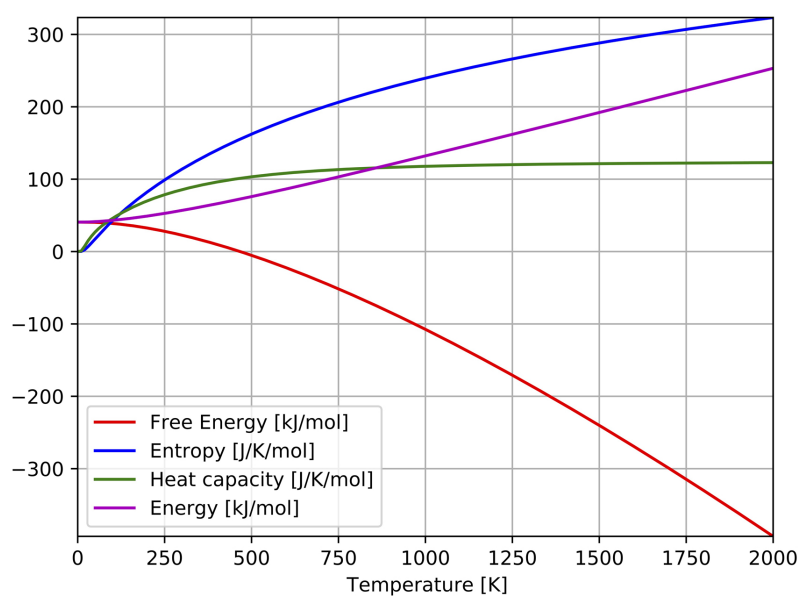

Figure 6. Thermal Properties of A) $\mathrm{RbMgH}_{3}$, B) $\mathrm{RbCaH}_{3}$

\subsection{Hydrogen Storage Properties}

As for the hydrogen storage properties of $\mathrm{RbXH}_{3}$ compounds, it is investigated to this scope of work with the terms of gravimetric Hydrogen storage capacities and desorption temperatures which are given by Eq. 14 and 15 . While $\mathrm{RbBeH}_{3}$ is not stable dynamically, $\mathrm{RbMgH}_{3}$ and $\mathrm{RbCaH}_{3}$ is stable. That's why $\mathrm{RbBeH}_{3}$ was not calculated hydrogen storage properties and is not presented in this section.

$$
\begin{gathered}
C_{w t \%}=\left(\frac{\left(\frac{H}{M}\right) M_{H}}{M_{\text {Host }}+\left(\frac{H}{M}\right) M_{H}} \times 100\right) \% \\
\Delta H=\Delta S \times T
\end{gathered}
$$

The gravimetric Hydrogen storage capacities of $\mathrm{RbXH}_{3}$ compounds have been calculated using Eq. 14 where $\left(\frac{H}{M}\right)$ is hydrogen-to-material atom ratio, $M_{H}$ and $M_{\text {Host }}$ indicates molar masses of Hydrogen and host material, respectively. The goal is to see if there are possible uses and to get an idea in this context in their hydrogen applications. As is seen Table 4 calculated values of gravimetric hydrogen storage capacities are 1.325 for $\mathrm{RbMgH}_{3}$ and 1.165 for $\mathrm{RbCaH}_{3}$, respectively. Among these structures the highest value of $C_{w t} \%$ has $\mathrm{RbMgH}_{3}$ while the lowest one is $\mathrm{RbCaH}_{3}$. As can be clearly seen in Eq. 14, $C_{w t \%}$ decreases as $\mathrm{X}$ increases since $\mathrm{C}_{\mathrm{wt} \%}$ depends on the atomic mass of $\mathrm{X}$ and also the number of Hydrogens in the unit cell is constant. To the best of our knowledge, the calculated $\mathrm{C}_{\mathrm{wt} \%}$ value is the first conclusion about possible hydrogen uses for these compounds and their place in Hydrogen applications. 
Table 4. Gravimetric Hydrogen Storage Capacities $\left(\mathrm{C}_{\mathrm{wt} \%}\right)$ and The Hydrogen Desorption Temperature of the $\mathrm{RbMgH}_{3}$ and $\mathrm{RbCaH}_{3}$ compounds

\begin{tabular}{ccc}
\hline Compounds & $\mathbf{C}_{\mathbf{w t} \%}$ & $\mathbf{T}\left({ }^{\circ} \mathbf{K}\right)$ \\
\hline $\mathrm{RbMgH}_{3}$ & 1.325 & 456.220 \\
$\mathrm{RbCaH}_{3}$ & 1.165 & 344.010 \\
\hline
\end{tabular}

Moreover, the desorption temperature, defined as the temperature involved to bring out $\mathrm{H}$ from structure, and it can be calculated with given by Eq. 15 . This value is remarkable about concerning the potential of the material, whether to be used in applications. In Eq. 15, $\Delta H$ indicates calculated formation enthalpy, $\Delta S$ symbolizes the entropy conversion of $\mathrm{H}$ at $1 \mathrm{~atm}$, which is $130.7 \mathrm{~J} / \mathrm{molK}$ and $\mathrm{T}$ shows desorption temperature. Calculated results are presented in Table 4. Although $\mathrm{RbCaH}_{3}$ is one of the most studied compound in literature and also one of the component of this study, the gravimetric hydrogen storage capacities and desorption temperature for neither $\mathrm{RbMgH}_{3}$ nor $\mathrm{RbCaH}_{3}$ are presented in previously studies for comparison. To the best our knowledge, the gravimetric hydrogen storage capacities and desorption temperature are the first calculated quantities for these compounds. As can be seen in Table 4, when the hydrogen desorption temperatures for these compounds are compared among themselves, they diminish as the $\mathrm{X}$ atom changes.

\section{Conclusion}

Consequently, in this work $\mathrm{RbXH}_{3}$ Perovskite type Hydrides compounds has been investigated via VASP based on DFT using the Generalized Gradient Approximation (GGA) with Perdew-Burke-Ernzerhof (PBE) functional. In this work found lattice parameter is 3.760, 4.118 and $4.545 \AA$ for $\mathrm{RbBeH}_{3}, \mathrm{RbMgH}_{3}$ and $\mathrm{RbCaH}_{3}$ respectively. For $\mathrm{RbCaH}_{3}$, a lattice parameter was calculated too close to the experimental value of $4.547 \AA$ presented in the study of Sato et al. For this reason, it is considered that our study is good represented $\mathrm{RbCaH}_{3}$ than the other theoretical works. Moreover the density of $\mathrm{RbCaH}_{3}$ was calculated with $0.779 \%$ margin of error from the other study presented. While $\mathrm{B}_{0}$ value is calculated with $0.885 \%$ margin of error from an experimental study, it was calculated too close to the theoretical one. As for the calculated formation enthalpy and gravimetric hydrogen storage capacity of $\mathrm{RbCaH}_{3}$, to the best our knowledge, this investigate is the first study of presented these values. As for the other two, to the best our knowledge, this work is the first study of these compounds.

Calculated values of gravimetric hydrogen storage capacities are between 1.325 and $1.165 \mathrm{wt} \%$, here the highest value belong to $\mathrm{RMgH}_{3}$, the lowest one is $\mathrm{RbCaH}_{3}$. These calculated capacities can be made a sensation about the potential of hydrogen applications and to possible uses. As for anisotropic properties of these compounds, thanks to elate, it has been determined to anisotropic properties of these molecules. When it investigated to their anisotropic properties, Poisson Ratio of $\mathrm{RbBeH}_{3}$ stands out dramatically. Since that value is outside -1 to 0.5 , it can be mentioned that deformation will be permanent for this material.

The electronic properties of these compounds are found to be semiconductor behaviour. In this respect, while $\mathrm{RbBeH}_{3}$ and $\mathrm{RbMgH}_{3}$ have an indirect bandgap with $0.274 \mathrm{eV}$ and $2.209 \mathrm{eV}, \mathrm{RbCaH}_{3}$ has a direct bandgap with $3.274 \mathrm{eV}$, it is found. In addition to, it is looked at band structure plots of these compounds, while the conduction of $\mathrm{RbBeH}_{3}$ and $\mathrm{RbMgH}_{3}$ is thought to be mainly over the $p$-band, $\mathrm{RbCaH}_{3}$ is thought to be over the mainly $d$-band.

The vibrational properties, $\mathrm{RbBeH}_{3}$ was unstable due to soft mode and the other two was stable.

As can be understood phonon dispersion curves and corresponding density of states, $\mathrm{Rb}$ is dominant between $0-5 \mathrm{THz}$ frequencies, Be atom is dominant at 5-15 $\mathrm{THz}$ and $\mathrm{H}$ atom is dominant between 20-30 THz for $\mathrm{RbBeH}_{3}$. As for $\mathrm{RbMgH}_{3}, \mathrm{Rb}$ is 
overpowering in the same area, $\mathrm{Mg}$ is overpowering at $5-10 \mathrm{THz}, \mathrm{H}$ is overpowering at $15-35 \mathrm{THz}$ and there is a gap more than $10 \mathrm{THz}$ between $\mathrm{Mg}$ and $\mathrm{H}$ it can be seen. In $\mathrm{RbCaH}_{3}$, the branches that are separate in $\mathrm{RbMgH}_{3}$ are intertwined for $\mathrm{Rb}, \mathrm{Mg}$ and $\mathrm{H}$ at low frequencies, and after a gap of the same length, $\mathrm{H}$ is dominant at high frequencies. As to thermal properties, throughout the temperature 0 to $2000^{\circ} \mathrm{K}$, it can be obviously seen that free energy was decreased while entropy and energy was increased besides that the heat capacity reaches a constant called as Dulong-Petit limit around the $700{ }^{\circ} \mathrm{K}$.

Author Contributions: Conceptualization, writing-original draft, methodology, writing-review and editing, visualization, supervision, formal analysis, investigation, validation, Ülkü BAYHAN; conceptualization, methodology, software, writing—original draft preparation İnanç YILMAZ. All authors have read and agreed to the published version of the manuscript.

Acknowledgments: This study was carried out with products from 0362-NAP16 and 0147-NAP12 projects financed and supported by Burdur Mehmet Akif Ersoy University. The authors, therefore, thanks to the Burdur Mehmet Akif Ersoy University for technical and financial support and NanoSim Laboratory for use of HPC Systems. The authors thanks to Prof. Dr. Yasemin ÖZTEKIN ÇİFTÇİ at Gazi University due to their contribution.

Conflicts of Interest: The authors declare that they have no competing financial interest or personal relationships that could have appeared to influence the work reported in this paper.

\section{Appendix A}
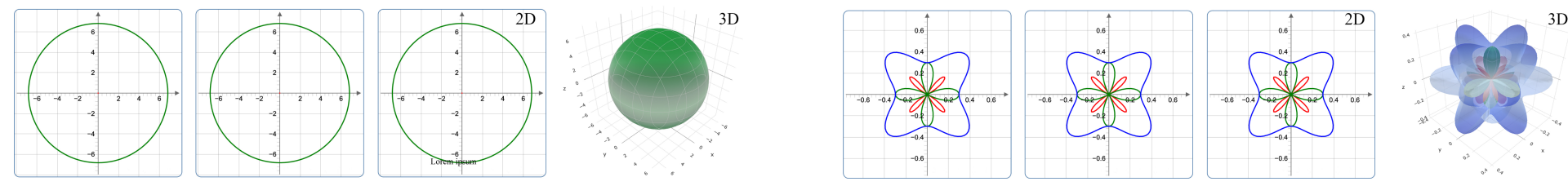

A) Linear Compressibility of $\mathrm{RbBeH}$

B) Poisson's Ratio of $\mathrm{RbBeH}_{3}$
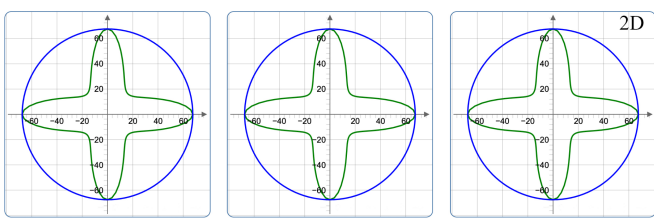

C) Shear Modulus of $\mathrm{RbBeH}_{3}$
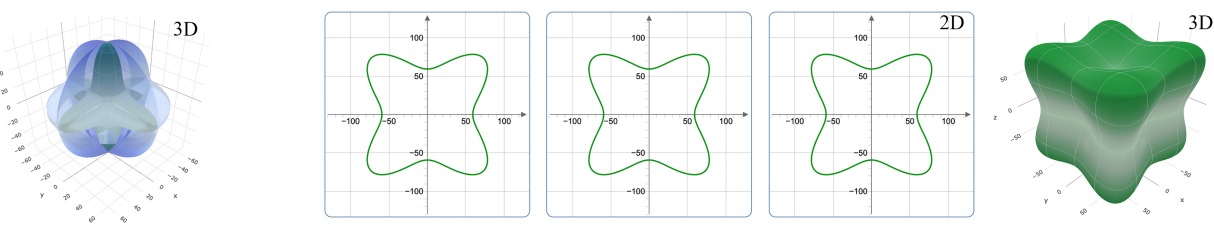

D) Young Modulus of $\mathrm{RbBeH}_{3}$

Figure 1. A) Linear Compressibility, B) Poisson's Ratio, C) Shear Modulus and D) Young's Modulus of $\mathrm{RbBeH}_{3}$
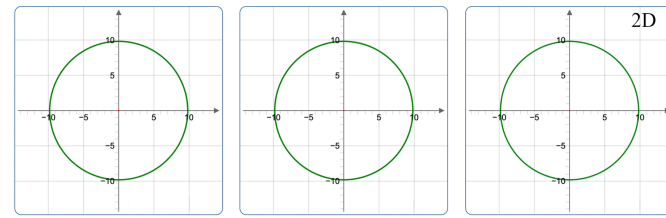

A) Linear Compressibility of $\mathrm{RbMgH}_{3}$
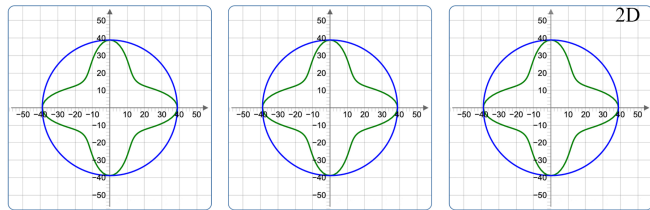

C) Shear Modulus of $\mathrm{RbMgH}_{3}$
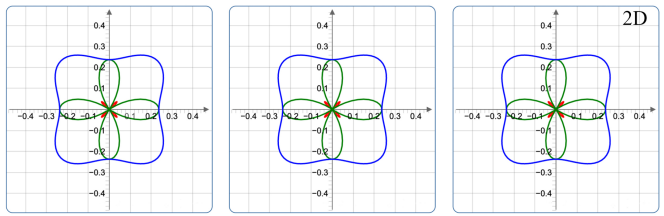

B) Poisson's Ratio of RbMgH
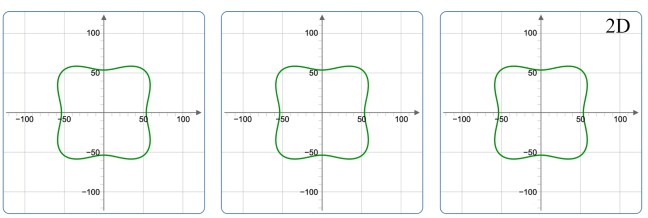

D) Young Modulus of $\mathrm{RbMgH}$

Figure 2. A) Linear Compressibility, B) Poisson's Ratio, C) Shear Modulus and D) Young's Modulus of $\mathrm{RbMgH}_{3}$

\section{References}

1. Gencer A.; Surucu G. Investigation of structural, electronic and lattice dynamical properties of $\mathrm{XNiH}(\mathrm{X}=\mathrm{Li}, \mathrm{Na}$ and $\mathrm{K})$ perovskite type hydrides and their hydrogen storage applications. International Journal of Hydrogen Energy, 2019, 44, 15173-15182. 
2. Candan A.; Kurban M. Electronic structure, elastic, and phonon properties of perovskite-type hydrides $\mathrm{MgXH} 3(\mathrm{X}=\mathrm{Fe}, \mathrm{Co}) \mathrm{for}$ hydrogen storage. Solid State Commications, 2018, 281, 38-43

3. Li, Y.; Chung, J. S.; Sung G. K. First-Principles Computational Screening of Perovskite Hydrides for Hydrogen Release. ACS Combinatorial Science, 2019, 21, 736-742.

4. Alam A.; Adel F.; Matar, S. F.; Ouaini, N. Nickel induced iono-covalent character of hydrogen in $\mathrm{RbMgH}_{3}$ from first principles. Chemical Physics Letters, 2011, 516, 174-176.

5. Myers, W. R.; Wang, L. W.; Richardson, T. J.; Rubin, M. D. Calculation of thermodynamic, electronic, and optical properties of monoclinic $\mathrm{Mg}_{2} \mathrm{NiH}_{4}$. Journal of Applied Physics, 2002, 91, 4879-4885.

6. Walker, G. 1 - Hydrogen storage technologies Solid-State Hydrogen Storage; Gavin Walker; Woodhead Publishing: Sawston, Cambridge, United Kingdom 2008; pp. 3-17.

7. Vajeeston, P.; Ravindran, P.; Fjellvåg, H. Structural phase stability studies on $\mathrm{MBeH}_{3}(\mathrm{M}=\mathrm{Li}, \mathrm{Na}, \mathrm{K}, \mathrm{Rb}, \mathrm{Cs})$ from density functional calculations. Inorganic Chemistry, 2008, 47, 508-514

8. Santhosh, M.; Kanagaprabha, S.; Rajeswarapalanichamy, R.; Sudha Priyanga, G.; Iyakutti, K. First principles study of structural stability, electronic structure and mechanical properties of alkali beryllium hydrides $\mathrm{ABeH}_{3}(\mathrm{~A}=\mathrm{K}, \mathrm{Rb}, \mathrm{Cs})$. Journal of Physics and Chemistry of Solids, 2015, 81, 34-39.

9. Shinzato, Y.; Yukawa, H.; Morinaga, M.; Baba, T.; Nakai, H. Energy density analysis of the chemical bond between atoms in perovskite-type hydrides. Journal of Alloys and Compounds, 2007 446-447, 96-100.

10. Ghebouli, B.; Ghebouli, M. A.; Fatmi, M. First-principles study of structural, elastic, electronic and optical properties of perovskites $\mathrm{XCaH}_{3}(\mathrm{X}=\mathrm{Cs}$ and $\mathrm{Rb})$ under pressure. Solid State Sciences, 2010, 12, 587-596.

11. Vajeeston, P.; Ravindran, P.; Fjellvåg, H. Structural investigation and thermodynamical properties of alkali calcium trihydrides. Journal of Chemical Physics, 2010, 132, 114504.

12. Lamichhane, S.; Aryal, B.; Kaphle, GC.; Adhikari, NP. Structural and electronic properties of perovskite hydrides $\mathrm{ACaH}_{3}(\mathrm{~A}=\mathrm{Cs}$ and $\mathrm{Rb})$. Bibechana, 2015, 13 94-99.

13. Gingl, F.; Vogt, T.; Akiba, E.; Yvon, K. Cubic $\mathrm{CsCaH}_{3}$ and hexagonal $\mathrm{RbMgH}_{3}$ : New examples of fluoride-related perovskite-type hydrides Journal of Alloys and Compounds, 1999, 282, 125-129.

14. Sato, T.; Noréus, D.; Takeshita, H.; Häussermann, U. Hydrides with the perovskite structure: General bonding and stability considerations and the new representative $\mathrm{CaNiH}_{3}$. Journal of Solid State Chemistry, 2005, 178, 3381-3388.

15. Kresse, G.; Furthmüller, J. Efficient iterative schemes for ab initio total-energy calculations using a plane-wave basis set. Physical Review B, 1996, 54, 11169-11186.

16. Momma, K.; Izumi, F. VESTA3 for three-dimensional visualization of crystal, volumetric and morphology data. Journal of Applied Crystallography , 2011, 44, 1272-1276

17. Kresse, G.; Joubert, D. From ultrasoft pseudopotentials to the projector augmented-wave method. Physical Review B - Condensed Matter and Materials Physics, 1999, 59, 1758-1775.

18. Blöchl, P. E. Projector augmented-wave method. Physical Review B, 1994, 50, 17953-17979.

19. Perdew, J. P.; Burke, K.; Ernzerhof, M. Generalized Gradient Approximation Made Simple. Physical Review Letters, 1996, 77, 3865-3868.

20. Methfessel, M.; Paxton, A. T. High-precision sampling for Brillouin-zone integration in metals Physical Review B, 1989, 40, 3616-3621.

21. Gaillac, R.; Pullumbi, P.; Coudert, F. ELATE: an open-source online application for analysis and visualization of elastic tensors. Journal of Physics: Condensed Matter, 2016, 275201.

22. Marmier, A.; Lethbridge, Z. A.D.; Walton, R. I.; Smith, C. W.; Parker, S. C.; Evans, K. E.; ElAM: A computer program for the analysis and representation of anisotropic elastic properties. Computer Physics Communications, 2010, 181, $2102-2115$.

23. Togo, A.; Oba, F.; Tanaka, I. First-principles calculations of the ferroelastic transition between rutile-type and $\mathrm{CaCl}_{2}$-type $\mathrm{SiO}_{2}$ at high pressures. Physical Review B - Condensed Matter and Materials Physics, 2008, 78, 134106.

24. Gonze, X.; Lee, C. Dynamical matrices, Born effective charges, dielectric permittivity tensors, and interatomic force constants from density-functional perturbation theory. Physical Review B, 1997, 55, 10355-10368.

25. Birch, F., Finite Elastic Strain of Cubic Crystals. Physical Review, 1947, 11, 809-824

26. Wang, J.; Yip, S.; Phillpot, SR.; Wolf, D. Crystal instabilities at finite strain. Physical review letters, 1993, 71, 4182.

27. Ranganathan, S. I. Ostoja-Starzewski, M. Universal Elastic Anisotropy Index. Physical Review Letters, 2008, 101, 055504.

28. Hamioud F.; Mubarak A. A. Structural, elastic and optoelectronic properties of the hydrogen based perovskite compounds: Ab-initio study. Chinese Journal of Physics, 2018, 56, 1-9

29. Chen, X.; Niu, H.; Li, D.; Li, Y. Modeling hardness of polycrystalline materials and bulk metallic glasses. Intermetallics, 2011, 19, 1275-1281. 